\title{
Food Safety Testing Technology Based on The Spectrophotometer and ARM
}

\author{
${ }^{\mathrm{a}}$ Jianjun Ding, ${ }^{\mathrm{b}}$ Xihua Wang \\ ${ }^{a}$ Physics and information school Jianghan University Wuhan, Hubei Province, P. R. China \\ ${ }^{b}$ Mechanical Engineering school Hubei University of Technology Wuhan, Hubei Province, P. R. China
}

\begin{abstract}
This paper describes using spectrophotometer and ARM in food safety detection system. Using the spectrophotometer as sensors will convert lead concentration and organophosphate pesticides concentration into optical signals. Data will be analyzed by ARM. This system can also complete photoelectric conversion, $\mathrm{A} / \mathrm{D}$ conversion functions and complete light signal processing effectively. The hardwares are low prices, costeffective, stable work, and easy to operate. This testing technology provides a reliable method for food safety detection.
\end{abstract}

Index Terms: Food Safety Detection;Spectrophotometer;ARM

(C) 2011 Published by MECS Publisher. Selection and/or peer review under responsibility of the International Conference on E-Business System and Education Technology

\section{Introduction}

The importance of food safety is of great significance for consumers. The state pays great attention to food safety issues and introduces the relevant laws and regulations[1]. As food safety testing items expanding and the improvement of detection accuracy continuously, food safety detection technology is facing new requirements now. So various of new testing methods appearing, many detection instruments being improved in practical constantly to meet the food safety detection technology requirements.

Pesticides are used in the production process of agricultural and this will produce pesticide residue. Although residues of pesticides are not very huge, general won't cause acute poisoning incidents happen, that it will still do harm to the health. When the aquatic breeding of water is contaminated by heavy metals that the heavy metal products can enter the body of aquatic during the process of growth gradually. When people eating tainted seafood, heavy metal will be transferred into their bodies. Because of the enrichment, long-term consumption of food containing heavy metals will lead to heavy metals poisoning in humans. Therefore, both the pesticide residue and the heavy metals residue are the key tests during the detection process. The food safety inspection technology bases on the spectrophotometer and the ARM can improve the detection accuracy

of these two tests and cut down the detection time. This will improve the level of food safety testing technology.

Corresponding author:

E-mail address: addjjd@126.com 


\section{The Whole System Design}

System flow chart is shown in Fig 1. Both ARM and spectrophotometer are used in the detection system. The material to be detected is treated into sample. Spectrophotometer will then convert organophosphorus pesticides and lead dosage to optical signals. Through the photoelectric conversion module, optical signals are converted into electrical signals. Because the output signals mix with clutter and the main signals are weak that the signals need to go through the filter and amplifier. After filtering and amplifying, signals are sent into the A/D converter module and generate digital signals which could be processed by ARM easily. In the process of analyzing data, ARM will call pre-prepared database and compare the input signals with the database information. In this way, it will check out whether the test samples are qualified or not. If the unqualified, it will send unqualified signal and alarm and show data exceed range.

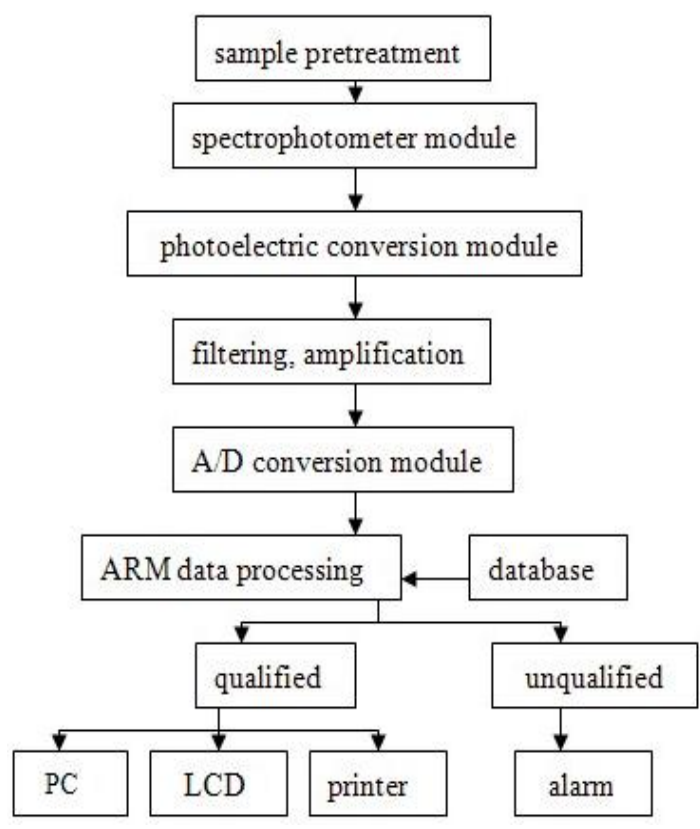

Fig 1. System Flow Chart

If qualified, data and test results will be displayed in the LCD screen and remind the operators that testing results will be sent to the PC or printed. After a series of processing, the final datas can be used to complete and update the database. This method can increase the number of sample database and improve the accuracy and stability of the system.

\section{Spectrophotometer Working Modules}

In this system, spectrophotometer is the most important sensor. The main function of the sensor is converting unvisible material concentration into optical signals. The detection method has the performances of high speed, high precision, stability and low cost. 


\section{1 Spectrophotometer Principle}

When light going through transparent liquid, substances containing in this liquid will absorb a certain wavelengths of the light and after going through the liquid, the energy of the light will be weakened[2]. The more reduction of the energy, the higher concentration of that material. So in this way, it can calculate the concentration of the substance containing in the liquid by calculating the reduction of the energy. Spectrophotometer is made basing on the principle. Using this kind of sensor, according to the operation specification strictly for inspection, it can still get a accurate test results in the case of small sample dose. What's more, the detection speed is fast enough.

\section{2 Spectrophotometer Module Workflow}

First, optical signal is generated by the light source. Usually the signal is white light. The light is gathered after entering the monochromator. The role of monochromator is that it can convert light into monochromatic light and in this way, there is any monochromatic for testing out corresponding material in the test[3]. Then the monochromatic light will go through the sample pool and reference pool. Sample pool is filled with liquid to be detected and reference pool is filled with the sample as the reference standard. Monochromatic light going through the sample pool and reference pool separately can eliminate the errors due to uncertain factors of environmental or instruments in test process effectively and this method, to a certain extent, can improve precision[4]. What's more, it also can ensure the sensors to collect signal accurately and validly and prepare for the later data processing.

\section{Photoelectric Conversion and ARM Data Analysis Module Principle}

Photoelectric conversion and ARM data analysis module are the key modules in this testing system for data analysis. The accuracy of the results, real-time detection process, the stability of the system state are largely depending on the the working conditions of the module.

\section{1 The Photoelectric Conversion Module Workflow}

After the monochromatic light output from the spectrophotometer, it is sent into electron converter. When optical signals are converted to electrical signals that the output signals are very weak and mix with the interferential signals. The signals can not be analyzed at that time. Therefore, they should be treated initially before the data analysis. First of all, it should filter out the clutter signals and preserve the main signals. Then isolate, amplify main signals. In this way, it can avoid the interference of other signals effectively. Finally, the system sends the main signals to A / D converter module. After the transformation, it can be receive as the digital signals by ARM.

\section{2 ARM Data Analysis Module Workflow}

Single chip microcomputer is used in traditional processing of data analysis. SCM is low cost and ease to program and use for the operators. However, with the growing types of test items, liquid elements more and more complex and the real-time detection requirements, the traditional SCM is no longer adapt to the requirements gradually. ARM7 is used in this system. The series processors support Windows, Linux and other operating systems with low power consumption and the command systems are compatible with ARM9 series and easy to upgrade[5]. The work flow of ARM processor is shown in Fig 2. 


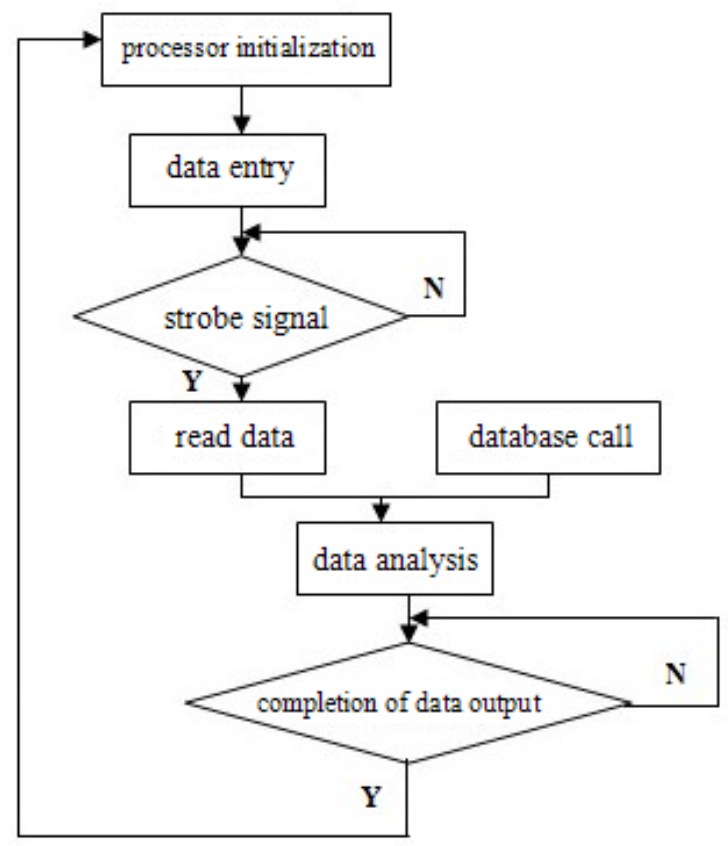

Fig 2. ARM processor work flowchart

First, initialize the processor. After the data inputting, the channel is gated and the system start to read the signals. ARM start to the call the database after the data getting into the processor. When the database being called to the ARM, the data from the test are analyzed and compared with the data from database. Finally, system exports the results and determines the output state and waits for the next round of data entry and analysis.

\section{Conclusion}

Spectrophotometer is easy to operate. If it is used with standard operation strictly that the result is very accurate. The treatment of pre-processing is relatively simple. What's more, it does not require large doses of samples and its power consumption is low. So, the cost of using spectrophotometer for organophosphate pesticides and heavy metals testing is relatively low. With the application of ARM7, the system anti-jamming ability is enhanced and the speed of data processing is increased and the performances of the detection accuracy and stability are improved[6]. The technology of spectrophotometer combining with ARM provides a safe and effective way for food safety testing.

\section{References}

[1] Jianjun Ding, Xihua Wang and Qicong Shen, “The Design of Piezoelectric Sensor Circuit In Food Safety Testing," Computer Knowledge and Technology(in Chinese), vol.6. Anhui, pp. 1234, 2010.

[2] Chao Chen, "Verification of The Problems Needing Attention in Visible Spectrophotometer," China Science and Technology Review(in Chinese). vol.4. Beijing, pp. 228, 2010. 
[3] Maofen Ma, Qiong Gong and Pinghui Li, "Application of Embedded System to the Design of Ultravioletvisible Spectrophotometer," Journal of NanYang Teachers College(in Chinese). vol.8. Henan, pp.34-35, 2009.

[4] Ke Li, "Application of High-Speed Signal Acquisition System Bases on DSP and ADS8364 in a NewType Spectrometer," Chinese Journal of Spectroscopy Laboratory(in Chinese). vol.26. Beijing, pp.16931694, 2009.

[5] Maohua Xiong and Zhenlun Yang, "The design and Development Application of ARM9 Embedded System,” (in Chinese) Tsinghua University Press. Beijing, pp.18, 2008. 